\title{
A general estimator of the primary cosmic ray energy with the ARGO-YBJ experiment
}

\section{Michele lacovacci ${ }^{a, b}$, Stefano Mastroianni ${ }^{b}$ on behalf of the ARGO-YBJ Collaboration}

a. Università "Federico II" di Napoli, Complesso Universitario di Monte Sant'Angelo, Via Cinthia, 80126 Napoli, Italy

b. INFN sez. di Napoli, Complesso Universitario di Monte Sant'Angelo, Via Cinthia, 80126

Napoli, Italy

E-mail: iacovacci@na.infn.it, mastroianni@na.infn.it,

The determination of the primary cosmic ray all-particle spectrum with ground-based air shower experiments usually depends on the assumed elemental composition and hadronic interaction model. Here we show that an energy estimator independent of the primary mass composition can be defined by means of shower parameters measured in the core region, as carried out in the ARGO-YBJ experiment. The energy resolution is $<10 \%$ above $100 \mathrm{TeV}$ and gets better with energy increasing. Being insensitive to the number of muons, this energy determination has only a weak dependence on the hadronic interaction model. The features of this energy estimator have been validated by extensive MC simulations and used in the analysis of the ARGO-YBJ data.

The 34th International Cosmic Ray Conference,

30 July- 6 August, 2015

The Hague, The Netherlands 


\section{Introduction}

The ARGO-YBJ detector [1, 2] is made of a single layer of Resistive Plate Chambers (RPCs) with a full coverage approach (sensitive area equal to $93 \%$ of the geometric one) located at 4300 m a.s.l.. The experiment was in stable data acquisition from November 2007 till February 2013 at Yangbajing Cosmic Ray Observatory. The digital readout of the signal (strip) provides a high spatial and temporal resolution in the shower front reconstruction for shower energy lower than a few hundred $\mathrm{TeV}$. To access higher energies and reach the knee region, the many $\mathrm{PeV}$, an analog readout [2] has been implemented by two electrodes of large dimensions, called Big Pad (BP), facing on the other side of the gas volume. Since December 2009 the analog readout of the RPC has been operating on the entire central carpet $\left(\sim 5800 \mathrm{~m}^{2}\right)$. In this configuration, the detector is capable of measuring particle density up to many thousands of particles per $\mathrm{m}^{2}$. Thanks to the analog readout, ARGO-YBJ is capable of providing a highly detailed picture of the charged particles in the core region of extensive air showers (EAS) .

The work presented here reports on the study of observables characterizing the EAS in the core region, namely the peak density or the maximum number of particles $P_{\max }$ on a BP, the truncated size $P_{10}$ which is the number of particles within 10 meters from the core, and the lateral age $s^{\prime}$ obtained by fitting the lateral distribution of particles with a Nishimura-Kamata-Greisen (NKG) like function. Thanks to the last two parameters, an energy estimator independent of the primary mass has be defined. Once validated on Montecarlo (MC) the energy reconstruction was applied to the ARGO-YBJ events and the all-particle spectrum was measured.

\section{MC Studies}

In the simulations we used CORSIKA[3, 4] with the code QGSjetII as hadronic interaction model, which is inspired by the theory of Regge-Gribov [5]. In general, the accuracy of the code is estimated in the order of $10 \%$ at the knee energies [6]. A considerable number of EAS (for proton, helium, $\mathrm{CNO}$ and iron nuclei respectively $1.5 \cdot 10^{7}, 3.0 \cdot 10^{6}, 2.0 \cdot 10^{6}$ and $2.0 \cdot 10^{6}$ ) with zenith angle $\theta<15^{\circ}$ and energy in the range $1-30000 \mathrm{TeV}$ was used. Then the ARGO-G code was used to simulate the response of the detector. To determine the core position the so-called Gravity Centre method has been applied to the 9 BPs centered around that one with the largest number of particles, or around $P_{\max }$. This reconstructed core position has been compared with the true core for each primary: for showers with truncated size $\left(P_{10}\right)$ greater than $10^{4}$ the difference is less than $2 \mathrm{~m}$ (iron showers). The event selection was made according to the following criteria:

- showers almost vertical, or $\theta<15^{\circ}$ (direction is determined by digital information);

- BP with the highest density of particles within the fiducial area, namely the 54 central clusters $\left(\sim 2400 m^{2}\right)$;

- reconstructed core position within the fiducial area.

In the selected events the mentioned quantities, $P_{\max }, P_{10}$ and $s^{\prime}$, characterizing the core region were studied. In Fig. $1 P_{\max }$ and $P_{10}$ are shown as a function of energy, in the range $100 \mathrm{TeV}-10$ $\mathrm{PeV}$, for the four simulated primaries. Both $\log \left(P_{\max }\right)$ and $\log \left(P_{10}\right)$ show a roughly linear trend with the increasing of $\log (E)$ and a decrease with the increasing of the primary mass.

About the lateral age $s^{\prime}$, it was obtained by fitting the lateral distribution of particles. Two functional 

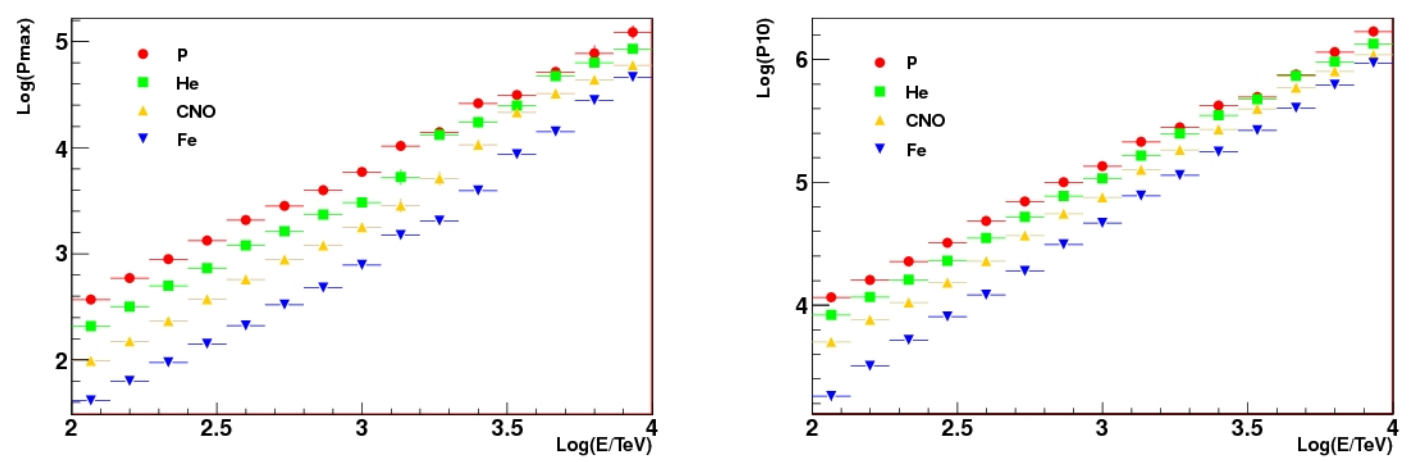

Figure 1: $P_{\max }$ (left) and $P_{10}$ (right) as a funtion of energy for the four primaries, see text for details.

forms were considered, the so-called Nishimura-Kamata-Greisen (NKG):

$$
f\left(r, s^{\prime}\right)=\frac{N_{e} C\left(s^{\prime}\right)}{2 \pi r_{M}^{2}} \cdot\left(\frac{r}{r_{M}}\right)^{s^{\prime}-2}\left(1-\frac{r}{r_{M}}\right)^{s^{\prime}-4.5}
$$

where $C\left(s^{\prime}\right)=\frac{\Gamma\left(4.5-s^{\prime}\right)}{\Gamma\left(s^{\prime}\right) \Gamma\left(4.5-2 s^{\prime}\right)}$

and its simplified, or polynomial, form (PL):

$$
f\left(r, s^{\prime}\right)=K \cdot\left(\frac{r}{r_{M}}\right)^{s^{\prime}-2}\left(1-\frac{r}{r_{M}}\right)^{s^{\prime}-4.5}
$$

where $K$ is a fitting parameter.

Only points within $0.5-10 \mathrm{~m}$ from the core position have been considered in the fit. For the parameter $r_{M}$, the Moliere radius, two values have been used, namely $30 \mathrm{~m}$ and $120 \mathrm{~m}$.

In Fig.2 (left) the distribution of $s^{\prime}$ obtained with the full NKG is compared to that obtained by the simplified form (PL), $r_{M}$ was set at $30 \mathrm{~m}$. The two distributions are equivalent, except for a peak of $s^{\prime}$ at 2.25, which shows up with the full NKG and is due to the divergence of $C\left(s^{\prime}\right)$ at that value. Considering that the functional form with $r_{M}=120 \mathrm{~m}$ is more sensitive to particles that fall at large distances from the core, while the one with $r_{M}=30 \mathrm{~m}$ is more sensitive to particles near the core, we have chosen to use the PL form and set $r_{M}=30 \mathrm{~m}$ in the analysis that follows.

In Fig. 2 on the right $s^{\prime}$ is shown as a function of energy, for different primaries, in the range 100 $\mathrm{TeV}-10 \mathrm{PeV}$. We may notice that, for energies above $200 \mathrm{TeV}, s^{\prime}$ shows a quite linear decreasing with $\log (E)$; at fixed energy $s^{\prime}$ increases with the primary mass.

Observing the behavior of $\log \left(P_{10}\right)$ and $s^{\prime}$ compared with $\log (E)$ and compared to the primary mass, we tried a combination of the two that was, at the same time, independent of the mass but strongly correlated with the energy; the following quantity:

$$
Y\left(s^{\prime}, P_{10}\right)=s^{\prime}+0.85 \cdot \log \left(P_{10}\right)
$$

satisfied the requirements. Fig. 3 shows $Y\left(s^{\prime}, P_{10}\right)$ as a function of $\log (E)$ for the four primaries. The symbols and the errors, for each primary, refer to mean value and the standard deviation of $Y$ in a given bin of energy. Then a linear fit has been performed for each primary according to:

$$
Y\left(s^{\prime}, P_{10}\right)=A+B \cdot \log (E)
$$



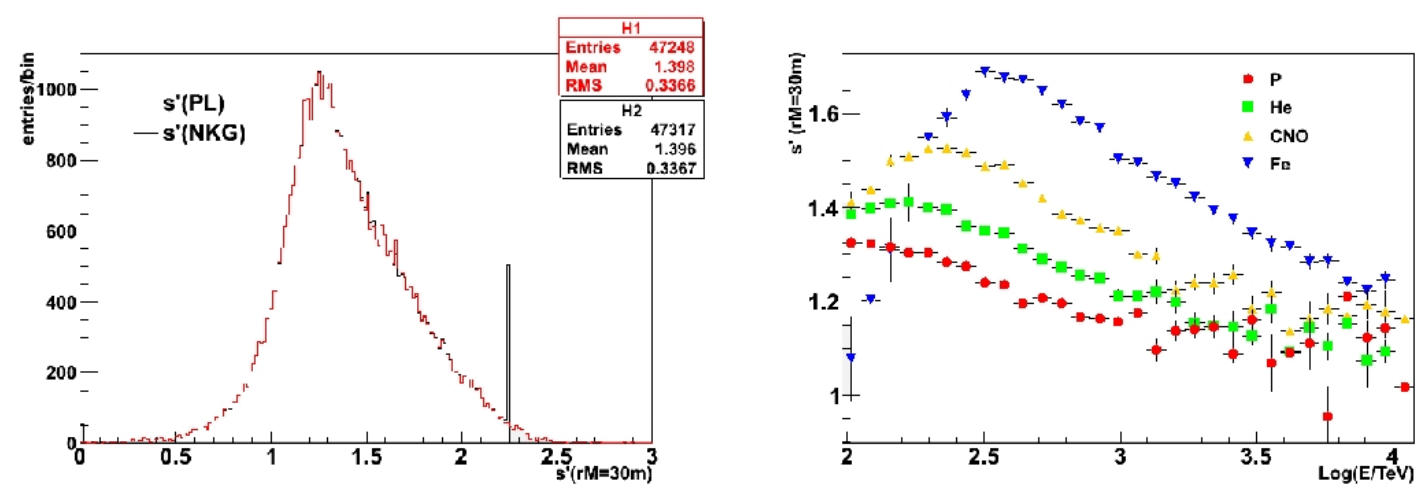

Figure 2: left, $s^{\prime}$ for NKG and PL function with $r_{M}=30 \mathrm{~m}$; right, $s^{\prime}$ as a function of energy for the four primaries - PL form and $r_{M}=30 \mathrm{~m}$

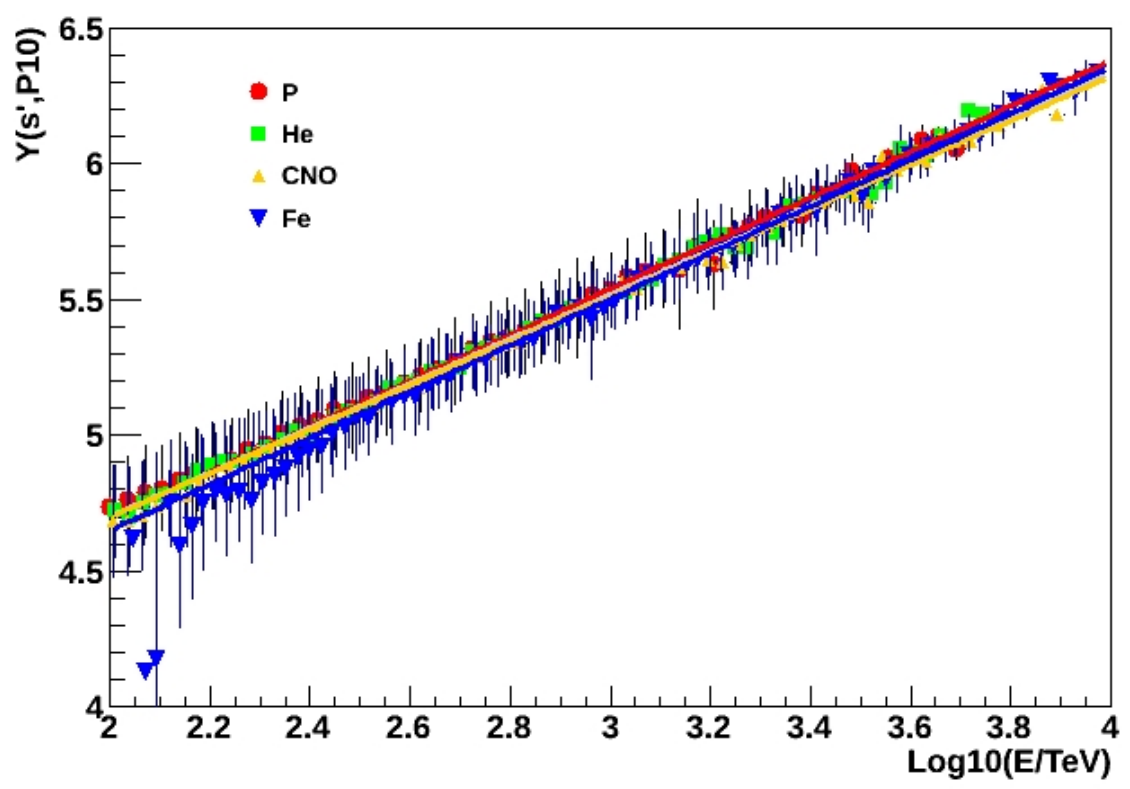

Figure 3: $Y\left(s^{\prime}, P_{10}\right)$ as defined in (2.3), as function of energy for the four primary particles. See text for details.

The values of A and B obtained for the different primaries (Tab.1) resulted to be fully compatible with each other, so that it was possible to define the reconstructed energy $E_{r e c}$ by the relation

$$
\log \left(E_{\text {rec }}\right)=\frac{Y\left(s^{\prime}, P_{10}\right)-A}{B}
$$

with $\mathrm{A}$ and $\mathrm{B}$ taken as the mean of the values found for the different primaries. In order to verify the quality of the energy estimator $E_{r e c}$, its spectrum was compared with the $E$ spectrum for proton, helium, CNO and iron primaries according to the Horandel model[7]. For this comparison, besides the selection criteria regarding zenith angle and core position, $\log \left(P_{10}\right)$ and $\log \left(P_{\max }\right)$ were taken in the ranges reported in Tab. 2 while for $s^{\prime}$ we required $0<s^{\prime}<2.5$. This condition is to select $s^{\prime}$ 


\begin{tabular}{|c|c|c|c|}
\hline Primary & $\log (E / \mathrm{TeV})$ & $\mathrm{A}$ & $\mathrm{B}$ \\
\hline PROTON & $2.2-3.5$ & $3.01 \pm 0.15$ & $0.842 \pm 0.046$ \\
\hline ELIUM & $2.2-3.5$ & $3.06 \pm 0.14$ & $0.818 \pm 0.044$ \\
\hline CNO & $2.3-3.5$ & $3.08 \pm 0.15$ & $0.811 \pm 0.049$ \\
\hline IRON & $2.5-3.5$ & $2.94 \pm 0.14$ & $0.852 \pm 0.046$ \\
\hline \hline$<>$ & & $3.02 \pm 0.15$ & $0.831 \pm 0.046$ \\
\hline
\end{tabular}

Table 1: A and B parameters, for each primary, as determined by the fit with (2.4). The second column shows the the energy interval where the fit was performed. In the last row are the means of the A and B values, which have been used for the energy reconstruction by (2.5).

\begin{tabular}{|c|c|c|c|c|}
\hline & $\rho_{\min }\left(m^{-2}\right)$ & $\rho_{\max }\left(m^{-2}\right)$ & $\log \left(P_{\max }\right)$ range & $\log \left(P_{10}\right)$ range \\
\hline G4 scale & $3 \pm 1$ & $900 \pm 22$ & {$[2.0,4.0]$} & {$[3.5,5.5]$} \\
\hline G1 scale & $12 \pm 3$ & $9000 \pm 200$ & {$[2.5,5.0]$} & {$[4.0,6.5]$} \\
\hline
\end{tabular}

Table 2: Average values of the minimum and maximum measurable particle density, namely $\rho_{\min }$ and $\rho_{\max }$, and relative errors for the two scales of operation [2] which the data refer to. In the 4.th and 5.th columns, are reported the ranges of $\log \left(P_{\max }\right)$ and $\log \left(P_{10}\right)$ considered in the analysis.

values that are meaningful from the physical point of view, in fact values outside of the range are typically caused by a lack of convergence of the fit to the lateral distribution.

The comparison allowed the estimate of the reconstruction efficiency. Two efficiencies have been considered, $E f f_{1}\left(E_{\text {rec }}\right)$ and $E f f_{2}(E)$. $E f f_{1}\left(E_{\text {rec }}\right)$ is defined as the ratio between the number of events within a bin of $E_{r e c}$, after the cuts on $P_{\max }, P_{10}$ and $s^{\prime}$ were appplied, and the number of generated events with energy $E$ within that bin; $E f f_{2}(E)$, instead, is the ratio between the number of events within a bin of $E$, downstream of the applied cuts, and the number of generated events with energy $E$ within that bin. While $E f f_{2}(E)$ shows the effect of the applied cuts, $E f f_{1}\left(E_{r e c}\right)$ shows the effect of both the cuts and the energy reconstruction.

However, to realistically define the efficiencies, the limited dynamic range of the detector had to be taken into account. In fact, as discussed in detail in [2], the readout electronics of the analog detector can measure particle densities ranging from a minimum density $\rho_{\min }$ up to a maximum density $\rho_{\max }$. The two parameters $\rho_{\min }$ and $\rho_{\max }$ affect the estimate of $P_{10}$ and $s^{\prime}$ which are used to the energy reconstruction. The values of $\rho_{\min }$ and $\rho_{\max }$ slightly vary from channel to channel and their average values and relative r.m.s. are shown in Tab.2 for the two scales of operation which the data used in this analysis refer to.

Then, similarly to what done in case of no limitation (infinite dynamic range), the whole procedure of energy reconstruction has been repeated on MC events where particle density lower than $\rho_{\text {min }}$ were set to 0 , while particle densities higher than $\rho_{\max }$ were taken as $\rho_{\max }$. For each event $P_{10}$ and $s^{\prime}$ were determined and $Y\left(s^{\prime}, P_{10}\right)$ calculated. Still using (2.4) the A and B parameters were determined for each primary and for both G4 and G1 scales. The parameter values so obtained resulted to be compatible with those previously found for infinite dynamic range. To determine the reconstructed energy $E_{\text {rec }}$ still the equation (2.5) has been used with A and B taken as the mean values among 

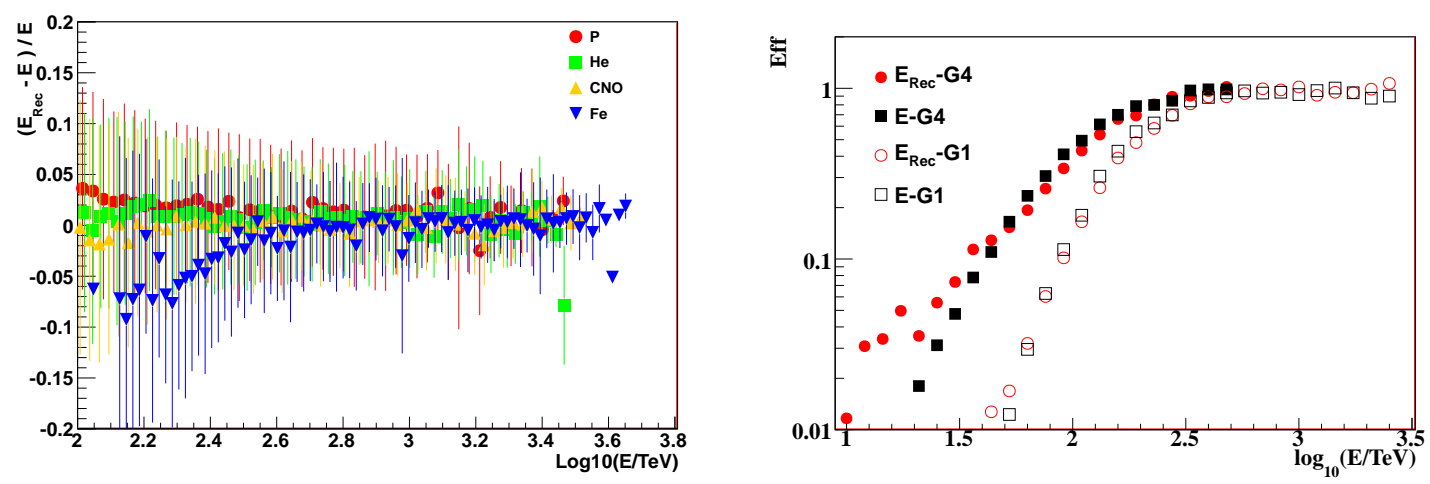

Figure 4: Left panel: Energy resolution of the method or percentage difference between the reconstructed energy $E_{\text {rec }}$ and the thrue energy $\mathrm{E}$ for the different primaries . Right panel: Mean efficiencies for the G4 and G1 scales, see text for details;

\begin{tabular}{|c|c|c|c|}
\hline Period & scale & Number of events & Selected events $(\theta-$ core $)$ \\
\hline 2010, day 195-229 & G4 & $16.77 \cdot 10^{6}$ & $1.14 \cdot 10^{6}$ \\
\hline 2010, day 270-365 & G1 & $58.16 \cdot 10^{6}$ & $3.90 \cdot 10^{6}$ \\
\hline
\end{tabular}

Table 3: Data sample used in this analysis. Number of events is for $P_{\max }>10$.

the four primaries. The energy resolution of the method, or the percentage difference between the reconstructed energy $E_{r e c}$ and the thrue energy E, is reported vs the energy for different primaries in Fig.4 (left). The behavior is similar for all primary components, that means the experimental energy resolution is independent of the composition model. The energy resolution is $<10 \%$ above $100 \mathrm{TeV}$ and gets better with energy increasing: above $300 \mathrm{TeV}$ gets below 5\% independent of the primary mass. As check of consistency we reconstructed the all-particle spectrum of the MC events, which actually were generated according to the Horandel model but with no cutoff for any component. The efficiency of reconstruction was taken as the weighted average of the efficiencies of the single components, the weight of each component being the flux of the component in the model (Fig.4, right). The results show a very good agreement, for both scales of operation, between the generated all particle spectrum and that one reconstructed by the energy estimator $E_{\text {rec }}$.

\section{Analysis of the ARGO-YBJ data}

The data sample used in this analysis is summarized in Tab.3, the data have been acquired by both scales G4 and G1, that means they cover the energy range $100 \mathrm{TeV}$ - many PeV. Before applying the selection on zenith angle and core location, we proceeded with the data quality check in order to have a data sample as much as possible not contaminated by spurious events mainly due to malfunctioning of electronic channels. Then the selection on zenith angle and core location was applied, as already explained for MC events at pag.2, and a comparison data-MC was carried out in order to check how well the MC reproduces the distributions of $P_{10}$ and $s^{\prime}$. The comparisons for $P_{10}$ and $s^{\prime}$ are shown in Fig.5, here the MC refers to the detector with infinite dynamic range. The primary fluxes are always according to [7]. The $P_{10}$ distribution for scale G4 shows an excellent 

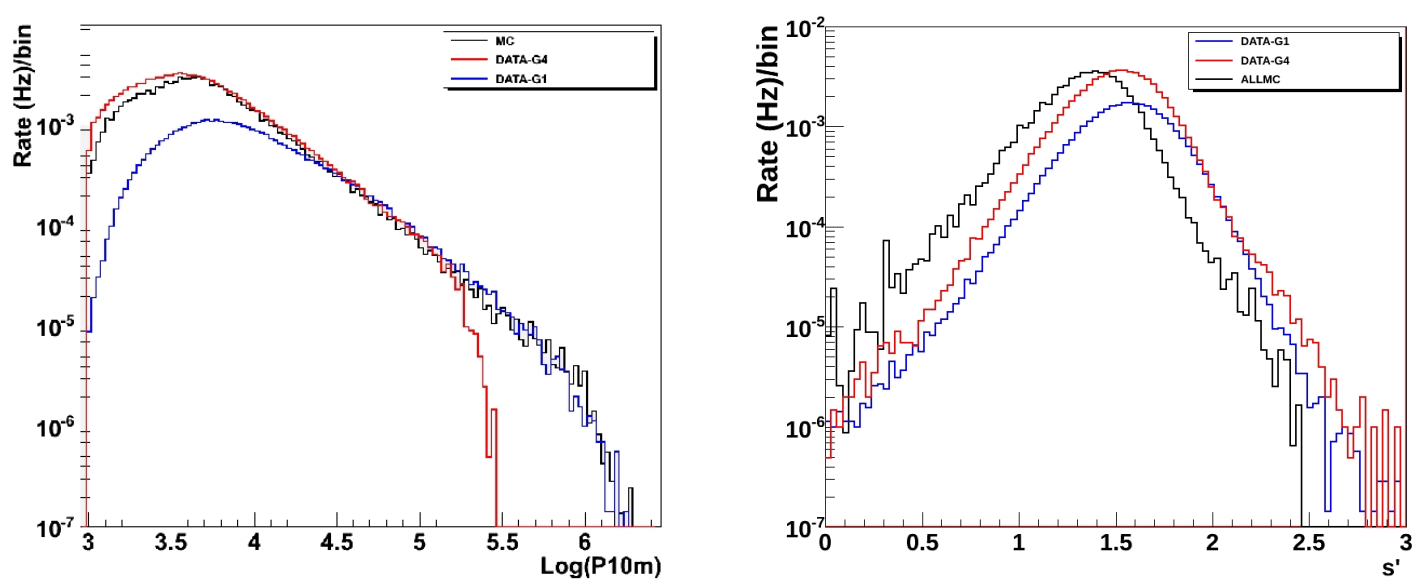

Figure 5: Distribution of the truncated size $P_{10}$ (left) and local age $s^{\prime}$ (right) for MC events and data (scales G4 and G1).

\begin{tabular}{|l|c|c|}
\hline & rate $_{\text {failure }}$-dati & rate $_{\text {failure }}-\mathrm{MC}$ (only proton) \\
\hline G4 scale & $0.09 \%$ & $0.07 \%$ \\
\hline G1 scale & $0.05 \%$ & $0.04 \%$ \\
\hline
\end{tabular}

Table 4: Failure rate of the fitting procedure for $s^{\prime}$ determination. Data are from both G4 and G1 scales.

agreement with the MC expectation, from values as low as $\log \left(P_{10}\right) \sim 3.5$ up to $\log \left(P_{10}\right) \sim 5.3$ where the saturation shows up. For scale G1 $P_{10}$ is quite inefficient for $\log \left(P_{10}\right)<\sim 4.3$, while the saturation effect is less pronounced. For each scale $P_{10}$ has a suited range where there is a very good consistency between data and MC. The Fig.5, on the right, reports the $s^{\prime}$ distribution for MC and data, scales G4 and G1. The failure rate of the fitting procedure for the $s^{\prime}$ determination is reported in Tab.4 for both the data samples (G1 and G4) and the MC sample (only protons). The difference between the distributions of the scales G4 and G1 is naturally explained by the different energy intervals in which G1 and G4 operate. Importantly the data show a difference of $s^{\prime}$ of about 0.150.20 with respect to MC. Several tests have been conducted to explain this difference, for example by the use of different $r_{M}$ in the functional form or through a different range of fit. However no satisfactory explanation was found yet for it. Such systematic could in fact be explained by a different model of composition and/or of hadronic interaction. After determination of $E_{\text {rec }}$ and correcting for the efficiencies shown in Fig.4, the left, and for the exposure, the all-particle spectrum measured by ARGO-YBJ was obtained. Given the number of events available in the two data samples (G1 and G4) we choose to work with energy intervals defined by $\Delta \log (E / T e V)=0.1$. This choice ensures a number of events sufficient to consider negligible the statistical errors. The flux measured by ARGO-YBJ is shown in Fig.6. To better highlight the structures, the spectra were multiplied by $E^{2.6}$. The dashed band corresponds to our systematic uncertainties which were estimated, as already explained, by using in the energy reconstruction the two extreme cases: the $\mathrm{A}$ and $\mathrm{B}$ values for protons and iron nuclei. The systematic uncertainty thus obtained corresponds to about $15 \%$ on the entire range of measurement. You may notice that the G4 scale allows a 


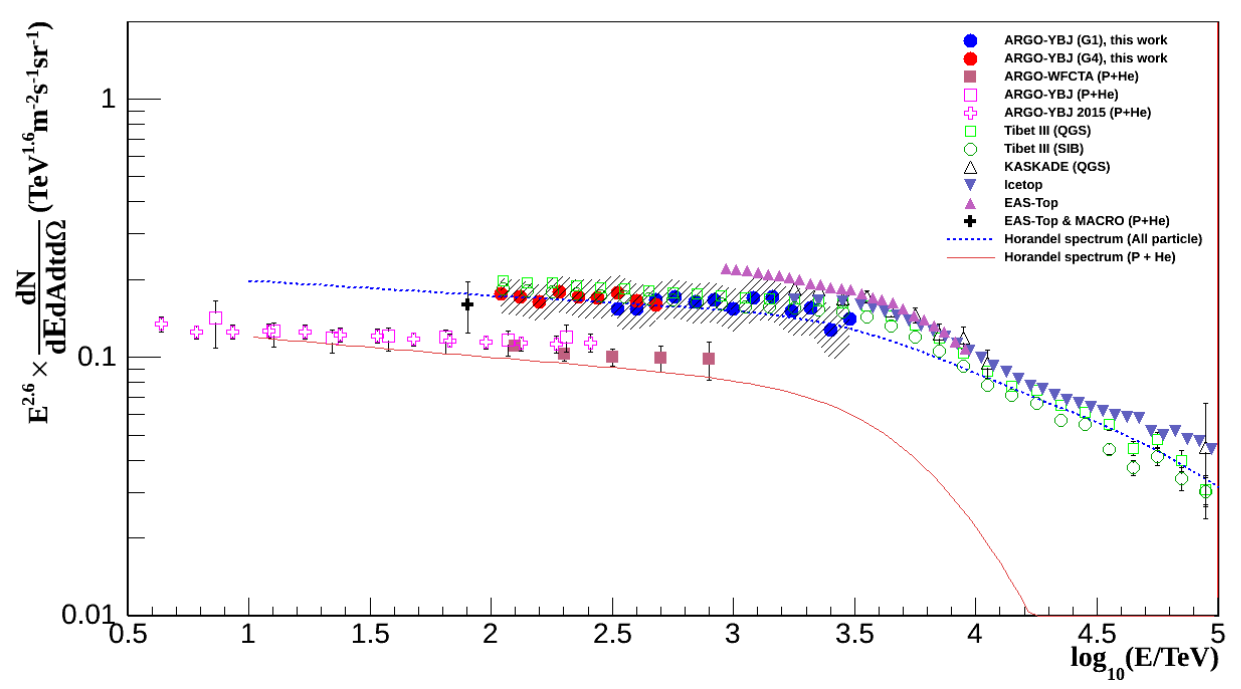

Figure 6: All-particle spectrum as measured by ARGO-YBJ in two overlapping energy ranges, namely 100-500 TeV (red filled circles) and 300-3000 TeV (blue filled circles), and comparison with the prediction of the Horandel model (dashed blue line). The all-particle spectra measured by KASCADE [11] , EAS-TOP [13], IceTop [14] and TIBET AS- $\gamma$ [15] are repoted for comparison. Also reported are the light component $(\mathrm{p}+\mathrm{He})$ as measured by ARGO-YBJ in the energy range of 3-300 TeV by using the digital readout $[8,9]$ (pink empty crosses and squares), as well the measurement performed by the hybrid detector consisting of the ARGO-YBJ carpet and the Cerenkov telescopes WFCTA from $100 \mathrm{TeV}$ up to $1 \mathrm{PeV}$ [10] (pink filled squares) and the EASTOP-MACRO measurement at $80 \mathrm{TeV}$ [12](pink empty crosses and squares).

good measurement of the energy spectrum from about $100 \mathrm{TeV}$ up to about $600 \mathrm{TeV}$, while the G1 scale from about $200 \mathrm{TeV}$ up to about $3 \mathrm{PeV}$. In such ranges of energy, in fact, the spectra measured by ARGO-YBJ with the two scales well overlap with each other and with the model's prediction. The Fig.6 also reports the light component ( $\mathrm{p}+\mathrm{He})$ as measured by ARGO-YBJ in the energy range of 3-300 $\mathrm{TeV}$ by using the digital readout $[8,9]$ (pink empty crosses and squares), as well as the measurement performed by the hybrid detector consisting of the ARGO-YBJ carpet and the Cerenkov telescopes WFCTA from $100 \mathrm{TeV}$ up to $1 \mathrm{PeV}$ [10] (pink filled squares ) and the EASTOP-MACRO measurement at $80 \mathrm{TeV}$ [12] (black filled crosses). The all-particle spectra measured by KASCADE [11], EAS-TOP [13], IceTop [14] and TIBET AS- $\gamma$ [15] are reported for comparison. We observe a very good agreement with the TIBET AS- $\gamma$ experiment, which is hosted at YBJ alongside ARGO-YBJ. Results given here, are consistent with the all-particle spectrum coming form an independent analysis of ARGO-YBJ data ( [16] and references therein). 


\section{Conclusions}

In this work we measured the all-particle spectrum of CR, between $100 \mathrm{TeV}$ and $3 \mathrm{PeV}$, analyzing the data of the ARGO-YBJ experiment. The data were collected with the analog readout system between 2010 and 2012. The study of MC events allowed the determination of a mass independent quantity strongly correlated to the energy, then the energy estimator $E_{r e c}$ was defined. The energy resolution is $<10 \%$ above $100 \mathrm{TeV}$ and gets better with energy increasing. The method, validated on simulated events, was applied to the data of ARGO-YBJ. Starting from the measured spectrum of $E_{r e c}$ and correcting for the overall efficiency, the all-particle spectrum of CR has been obtained. Systematic errors were evaluated in about $15 \%$, while the statistical errors are generally very low and below $5 \%$ for the very high energy points. The spectrum was compared with the expectation of the Horandel model and the results of other experiments, finding a good agreement with the model and good compatibility with other determinations of the spectrum. Results given here are consistent with the all-particle spectrum coming from independent analysis of ARGO-YBJ data.

\section{References}

[1] G. Aielli et al., NIMA, 562 (2006) 92

[2] B. Bartoli et al., Astrop. Phys., 67 (2015) 47

[3] D. Heck et al., Forschungszentrum Karlsruhe FZKA 6019 (1998)

[4] J. Knapp D.Heck, KfK 5196 B (1993)

[5] N.N. Kalmykov and S.S. Ostapchenko, Yad. Fiz. 56 (1993) 105

[6] S. P Swordy et al., Astropart. Phys. 18 (2002) 129

[7] Jorg R. Horandel, Astropart.Phys., 19 (2003) 193

[8] B. Bartoli et al., PHYS. REV. D 91 (2015)112017

[9] B. Bartoli et al., PHYS. REV. D 85 (2012) 092005

[10] B. Bartoli et al., Chin. Phys. C 38 (2014) 045001

[11] W.D.Apel et al.., Astropart. Phys. 47 (2013) 54

[12] M. Aglietta et al., Astropart. Phys. 21 (2004) 223

[13] M. Aglietta et al., Astropart. Phys. 21 (2004) 583

[14] M. G. Aartsen et al., PHYS. REV. D 88 (2013) 042004

[15] Annemori M. et al., Astrophys. J., 678 (2008) 1165

[16] A. D'Amone et al (ARGO-YBJ coll.), Proceedings of 34th ICRC, ID: 917, 2015 